\title{
A NEW ISOSCELES-TRIANGLE SOLUTION OF THE THREE BODY PROBLEM*
}

\author{
BY \\ DANIEL BUCHANAN
}

\section{INTRODUCTION}

The known isosceles-triangle solutions of the problem of three bodies are the periodic solutions in which two of the bodies have equal finite masses, while the third body moves in a straight line and remains equidistant from the other bodies. In my Chicago dissertation $†$ three cases of the isoscelestriangle solutions were discussed. In the first case the finite bodies move in a circle and the third body is infinitesimal. The solution of this case was first obtained by Pavaninił by means of elliptic integrals, and was later developed independently by MacMillan.§ The solution which was obtained in my dissertation is analogous to that obtained by MacMillan. In the second case, not treated by Pavanini and MacMillan, the finite bodies move in ellipses and the third body is infinitesimal. In the third case, all three bodies are finite. The period of the motion in case III is the same as that in case $I$, and the solution reduces to the solution of the first case when the third body becomes infinitesimal.

The present paper is an extension of the second case of my dissertation to that in which all three masses are finite. Periodic orbits are here shown to exist, and a practical method is given of computing them. These solutions have the same period as those of case II of my dissertation, and reduce to them when the third body becomes infinitesimal.

\section{The differential equations of motion}

Let $m_{1}$ and $m_{2}$ represent the two finite bodies of mass $m$, and let the third body be denoted by $\mu$. The origin of coördinates will be taken at the center of mass of the system. The plane perpendicular to the initial motion of $\mu$

* Presented to the Society (Chicago), December 28, 1914.

$\dagger$ Moulton's Periodic Orbits, Chap. X.

$\ddagger$ Annali di Matematica, ser. 3, vol. 13 (1907), pp. 179-202.

\$ A stronomical Journal, Nos. 625-626 (1911). 
will be taken as the $\xi \eta$-plane. Then let the coördinates of $m_{1}, m_{2}$, and $\mu$ be denoted by $\xi_{1}, \eta_{1}, \zeta_{1} ; \xi_{2}, \eta_{2}, \zeta_{2}$; and $\xi, \eta$, $\zeta$ respectively.

When $\mu=0$ the mean angular motion of the finite bodies is*

$$
n=\frac{k \sqrt{2 m}}{a^{3 / 2}},
$$

where $k$ is the gravitational constant and $a$ is the mean distance from $m_{1}$ to $m_{2}$. Now let the unit of mass be so chosen that $m=1 / 2$, and let the linear and time units be so determined that $k$ and $a$ are each unity. With the units thus chosen $n=1$.

We shall suppose that $\mu$ is started from the origin of coördinates at the initial time $t_{0}$ when $m_{1}$ and $m_{2}$ are at apsides of their orbits, which can be assumed, without loss of geometric generality, to lie on the $\xi$-axis. When expressed analytically, these initial conditions are

$$
\begin{gathered}
\xi=\eta=\zeta=\xi^{\prime}=\eta^{\prime}=\eta_{1}=\eta_{2}=\zeta_{1}=\zeta_{2}=0 \quad \text { at } t=t_{0}, \\
\xi_{1}\left(t_{0}\right)=-\xi_{2}\left(t_{0}\right)=\frac{a}{2}(1-e), \quad \xi_{1}^{\prime}\left(t_{0}\right)=-\xi_{2}^{\prime}\left(t_{0}\right), \quad \eta_{1}^{\prime}\left(t_{0}\right)=-\eta_{2}^{\prime}\left(t_{0}\right),
\end{gathered}
$$

where $e$ is the eccentricity of the ellipses in which $m_{1}$ and $m_{2}$ move when $\mu=0$. Under these initial conditions the orbits described by $m_{1}$ and $m_{2}$ will be symmetrical; that is,

$$
\xi_{1} \equiv-\xi_{2}, \quad \eta_{1} \equiv-\eta_{2}, \quad \zeta_{1} \equiv \zeta_{2} .
$$

On making use of (2) in the center of gravity equations, which are

we obtain

$$
\begin{gathered}
m_{1} \xi_{1}+m_{2} \xi_{2}+\mu \xi=0, \quad m_{1} \eta_{1}+m_{2} \eta_{2}+\mu \eta=0, \\
m_{1} \zeta_{1}+m_{2} \zeta_{2}+\mu \zeta=0
\end{gathered}
$$

$$
\xi \equiv \eta \equiv 0, \quad \zeta_{1}+\mu \zeta=0 .
$$

Hence the third body always remains on the $\zeta$-axis.

The differential equations of motion when simplified by (3) become

$$
\begin{aligned}
\xi_{1}^{\prime \prime} & =-\frac{\xi_{1}}{8 r^{3}}-\frac{\mu \xi_{1}}{\left[r^{2}+(1+\mu)^{2}\right.} \frac{\left.\zeta^{2}\right]^{3 / 2}}{\mu \eta_{1}} \\
\eta_{1}^{\prime \prime} & =-\frac{\eta_{1}}{8 r^{3}}-\frac{-2 m(1+\mu) \zeta}{\left[r^{2}+(1+\mu)^{2} \zeta^{2}\right]^{3 / 2}}, \\
\zeta^{\prime \prime} & =\frac{-2 m}{\left[r^{2}+(1+\mu)^{2} \zeta^{2}\right]^{3 / 2}},
\end{aligned}
$$

where $r^{2}=\xi_{1}^{2}+\eta_{1}^{2}$. When these equations have been integrated, the solu-

* Moulton's Celestial Mechanics, p. 142. 
tions for $\xi_{2}, \eta_{2}, \zeta_{1}$, and $\zeta_{2}$ can be obtained from (2) and (3). The numerical value of $m=1 / 2$ has been substituted in the first two equations of (4) but not in the last equation. The reason for this will appear in the next section.

On transforming to polar coördinates by the substitutions

$$
\xi_{1}=r \cos v, \quad \eta_{1}=r \sin v, \quad \zeta=z,
$$

the differential equations become

$$
\begin{aligned}
r^{\prime \prime}-r\left(v^{\prime}\right)^{2}+\frac{1}{8 r^{2}} & =-\frac{\mu r}{\left[r^{2}+(1+\mu)^{2} z^{2}\right]^{3 / 2}}, \\
r v^{\prime \prime}+2 r^{\prime} v^{\prime} & =0, \\
z^{\prime \prime} & =-\frac{2 m(1+\mu) z}{\left[r^{2}+(1+\mu)^{2} z^{2}\right]^{3 / 2}} .
\end{aligned}
$$

\section{The equations of variation}

For $\mu=0$, the first two equations of (5) reduce to those of the two-body problem, and their solutions are*

$$
\begin{aligned}
& r=r_{0}=\frac{a}{2}\left[1-e \cos n\left(t-t_{0}\right)+\frac{e^{2}}{2}\left\{1-\cos 2 n\left(t-t_{0}\right)\right\}+\cdots\right], \\
& v=v_{0}=n\left(t-t_{0}\right)+2 e \sin n\left(t-t_{0}\right)+\frac{5}{4} e^{2} \sin 2 n\left(t-t_{0}\right)+\cdots
\end{aligned}
$$

In these equations both $a$ and $n$ are unity, while $t_{0}$ can be chosen to be zero without loss of generality, but these numerical values are not substituted at present as we wish later to differentiate $r_{0}$ with respect to $t_{0}$.

The second equation of (5) admits the integral

$$
r^{2} v^{\prime}=c .
$$

When $\mu=0$ the third equation of (5) becomes

$$
z^{\prime \prime}=-\frac{2 m z}{\left[r^{2}+z^{2}\right]^{3 / 2}},
$$

where $r$ has the value $r_{0}$ obtained in (6). Now instead of putting $m=1 / 2$ in the numerator of (8), as was done in the first two equations of (4), we make the substitution

$$
m=m_{0}+\lambda,
$$

where $m_{0}$ is considered as a constant and $\lambda$ as a variable parameter. For the purpose of computation we must substitute $\lambda=m-m_{0}$ in the final results.

\footnotetext{
* Moulton's Celestial Mechanics, pp. 154, 155.
} 
It was shown in case II of my dissertation that periodic solutions of equation (8) exist, and are power series in $\lambda^{1 / 2}$ of the form

$$
\begin{aligned}
z_{0} & = \pm \lambda^{1 / 2}\left[\frac{-\sqrt{2}}{3 \sqrt{m_{0}}} \sin \sigma n\left(t-t_{0}\right)+\text { power series in } e\right]+\cdots \\
& = \pm \sum_{j=0}^{\infty} z_{2 j+1}\left(\lambda^{1 / 2}\right)^{2 j+1}
\end{aligned}
$$

where each $z_{2 j+1}$ is a power series in $e$ of the form

$$
z_{2 j+1}=\sum_{\kappa=0}^{\infty}\left[\sum_{l=0}^{j} \sum_{i=0}^{\kappa} c_{ \pm i, l, \kappa}^{2 j+1} \sin n\{(2 l+1) \sigma \pm i\}\left(t-t_{0}\right)\right] e^{\kappa} .
$$

The $c_{ \pm i, l, \kappa}^{2 j+1}$ are real constants and $\sigma$ is a power series in $e$ of the form

$$
\sigma=4 \sqrt{m_{0}}+\frac{3 \sqrt{m_{0}}\left(1-16 m_{0}\right)}{1-64 m_{0}} e^{2}+\cdots .
$$

As power series similar to $z_{0}$ in (9) occur frequently in the sequel, we shall call them $\lambda^{1 / 2}$-sine-series.

Since $\sigma$ is a continuous function of $e$, values of $e$ can be chosen so that $\sigma$ converges to a rational fraction $N / \nu$, where $N$ and $\nu$ are integers relatively prime. The period of the solutions (9) is then

$$
T=2 \nu \pi=\frac{2 N \pi}{\sigma}
$$

In $\$ 171$ of my dissertation,* a reference was given to show that the two solutions in (9) represent the same orbit if $\nu$ is even, but in the one the infinitesimal body is half a period ahead of its position in the other. If $\nu$ is odd the orbits for plus and minus are geometrically distinct. In the sequel we shall take only the plus sign in (9) as the solutions obtained from the minus sign are entirely analogous to those obtained when the plus sign is taken.

In order to find the solutions of (5) when $\mu$ is not zero, we make the substitutions

$$
r=r_{0}+p, \quad c=c_{0}+d \mu, \quad z=z_{0}+w,
$$

where $p$ and $w$ vanish with $\mu$, and $d$ is an arbitrary constant. From (7) it follows that

$$
c_{0}=r_{0}^{2} v_{0}^{\prime} \text {. }
$$

When $v^{\prime}$ is eliminated from the first equation of (5) by the integral (7), and the substitutions (11) are made, the terms in the first equation of (5) independent of $\mu$ are zero. Hence

$$
c_{0}^{2}=r_{0}^{\prime \prime} r_{0}^{3}+\frac{1}{8} r_{0} .
$$

\footnotetext{
* Moulton's Periodic Orbits.
} 
The differential equations determining $p$ and $w$ then become

$$
\begin{aligned}
p^{\prime \prime}+P(e, t) p & =P_{0}+P_{1} \mu+P_{2} \mu^{2}+\cdots, \\
w^{\prime \prime}+W(\lambda, e, t) w & =W_{0}+W_{1} \mu+W_{2} \mu^{2}+\cdots,
\end{aligned}
$$

where $P, W, P_{j}, W_{j}(j=0,1,2, \cdots)$ have the following properties:

(1) $P$ is a power series in $e$ whose coefficients are sums of cosines of multiples of $t$. In so far as the computations have been made, we have

$$
P(e, t)=1+6 e \cos t+e^{2}(3+12 \cos 2 t)+\cdots .
$$

(2) $W$ is a power series in $\lambda$ whose coefficients are power series in $e$. The coefficients of the various powers of $e$ have the form

$$
\sum_{k, l} c_{k, l} \cos (2 k \sigma \pm l) t
$$

where $k$ and $l$ are positive integers or zero, and $c_{k, l}$ are real constants. As power series of this kind occur frequently in the sequel we shall call them $\lambda$ cosine-series.

(3) $P_{0}$ is a power series in $p$ beginning with the term in $p^{2}$. The coefficients of the various powers of $p$ are power series in $e$ similar in form to $P$.

(4) $P_{j}(j=1,2,3, \cdots)$ are power series in $p$ and $w$. The coefficients of the terms in which $w$ occurs in even degrees are $\lambda$-cosine-series. The coefficients of the terms in which $w$ occurs in odd degrees are $\lambda^{1 / 2}$-sine-series.

(5) $W_{j}(j=0,1,2, \cdots)$ are power series in $p$ and $w$. The coefficients of the various powers of $p$ and $w$ are $\lambda^{1 / 2}$-sine-series if $w$ occurs in even degrees, and $\lambda$-cosine-series if $w$ oecurs in odd degrees.

If we put the left members in (13) equal to zero, we obtain the equations of variation, which are

$$
\text { (a) } \quad p^{\prime \prime}+P(e, t) p=0, \quad \text { (b) } \quad w^{\prime \prime}+W(\lambda, e, t) w=0 .
$$

\section{The solutions of the equations of variation}

The generating solutions of the equations of variation are $r_{0}$ and $z_{0}$ in (6) and (9) respectively. Each of these two generating solutions contains the arbitrary constant $t_{0}$, and hence one set of solutions of (14) is obtained by taking the first partial derivatives of $r_{0}$ and $z_{0}$ with respect to $t_{0} .^{*}$ One solution of the first equation of (14) is therefore

$$
\frac{\partial r_{0}}{\partial t_{0}}=-\frac{e}{2}[\sin t+e \sin 2 t+\cdots]=-\frac{e}{2} \psi(e, t),
$$

and as we multiply this solution later by an arbitrary constant, we may disregard the factor $-e / 2$.

* Poincaré's Les Méthodes Nouvelles de la Mécanique Céleste, vol. 1, Chap. IV. Moulton's Periodic Orbits, $\S \S 32,33$. 
When the generating solution contains an arbitrary constant one of the characteristic exponents of the solutions of the equations of variation is zero, and since the sum of the characteristic exponents is zero both these exponents are zero. In this case one of the solutions is a periodic function while the other is the sum of a periodic function and $t$ times the former solution. That is, the second solution of $(14 a)$ is

$$
p=\theta+A t \psi,
$$

where $\theta$ and $A$ are power series in $e$, the former having periodic coefficients, the latter having constant coefficients. In order to determine $\theta$ and $A$ we substitute $p=\theta+A t \psi$ in $(14 a)$ and integrate as power series in $e$. We choose the initial values $\theta(0)=1, \theta^{\prime}(0)=0$ and determine $A$ so that $\theta$ shall be periodic. The computation gives

$\theta=\cos t-e(3-2 \cos t-\cos 2 t)$

$$
\begin{gathered}
-e^{2}\left(6-\frac{23}{8} \cos t-2 \cos 2 t-\frac{9}{8} \cos 3 t\right)+\cdots, \\
A=\gamma e^{2}=[3+\text { power series in } e] e^{2} .
\end{gathered}
$$

Hence the two solutions of $(14 a)$ are $\psi$ and $\theta+\gamma e^{2} t \psi$. The determinant of these two solutions is a constant,* and its value at $t=0$ is

$$
D=-\theta(0) \psi^{\prime}(0)=-(1+2 e+\cdots) .
$$

This determinant is different from zero for $e=0$ and therefore remains different from zero for $|e|$ sufficiently small. Consequently the two solutions (15) constitute a fundamental set and the general solution of the first equation of (14) is

$$
p=A_{1} \psi+A_{2}\left[\theta+\gamma e^{2} t \psi\right],
$$

where $A_{1}$ and $A_{2}$ are the constants of integration. The functions $\psi$ and $\theta$ are periodic with the period $2 \pi$ and à posteriori with the period $T$.

Similarly, one of the solutions of the second equation of (14) is

$$
\frac{\partial z_{0}}{\partial t_{0}}=\lambda^{1 / 2} \phi(\lambda, e, t),
$$

where $\phi$ is a power series in $\lambda$ similar to a $\lambda$-cosine-series except that it has odd multiples of $\sigma$ in the arguments of the cosines instead of even multiples. By computation it is found that

$$
\phi(0)=\frac{\sqrt{2} \sigma}{3 \sqrt{m_{0}}}+\cdots, \quad \phi^{\prime}(0)=0 .
$$

The second solution of $(14 b)$ could be found in the same way as the second

\footnotetext{
* Moulton's Periodic Orbits, § 18.
} 
solution of (14a), but we shall determine it by a shorter method. We may consider $\lambda$ as an arbitrary constant and in this case the other solution of (14b) is

$$
\frac{\partial z_{0}}{\partial \lambda}=\left(\frac{\partial z_{0}}{\partial \lambda}\right)+\frac{\partial z_{0}}{\partial n} \frac{\partial n}{\partial \lambda},
$$

where $\left(\partial z_{0} / \partial \lambda\right)$ denotes that the differentiation is performed only in so far as $\lambda$ enters $z_{0}$ explicitly. Hence

$$
\left(\frac{\partial z_{0}}{\partial \lambda}\right)=\frac{\partial z_{0}}{\partial\left(\lambda^{1 / 2}\right)} \frac{\partial\left(\lambda^{1 / 2}\right)}{\partial \lambda}=-\frac{1}{\lambda^{1 / 2}} \chi,
$$

where $\chi$ is a power series in $\lambda$ with coefficients which are similar in form to the coefficients in a $\lambda^{1 / 2}$-sine-series. The values of $\chi(0)$ and $\chi^{\prime}(0)$ are found by computation to be

$$
\chi(0)=0, \quad \chi^{\prime}(0)=\left[\frac{\sqrt{2} \sigma}{6 \sqrt{m_{0}}}+\text { power series in } \lambda\right] .
$$

The partial derivative $\partial z_{0} / \partial n$ has the value

and since

$$
\frac{\partial z_{0}}{\partial n}=-\lambda^{1 / 2} t \phi
$$

$$
n=\frac{k \sqrt{2 m}}{a^{3 / 2}}=\frac{k \sqrt{2\left(m_{0}+\lambda\right)}}{a^{3 / 2}},
$$

then $\partial n / \partial \lambda=1$. Therefore the second solution of $(14 b)$ is

$$
-\frac{1}{\lambda^{1 / 2}}[\chi+\lambda t \phi] .
$$

The two solutions of $(14 b)$ then take the form

$$
\lambda^{1 / 2} \phi \text { and }-\frac{1}{\lambda^{1 / 2}}[\chi+\lambda t \phi],
$$

where $\phi$ and $\chi$ are periodic with the period $T$. As in the solution of (14a) we can omit the constants $\lambda^{1 / 2}$ and $-\lambda^{-1 / 2}$, and consider $\phi$ and $\chi+\lambda t \phi$ as the two solutions. The determinant of these solutions is a constant,* and at $t=0$ the value is

$$
\Delta=\phi(0)\left[\chi^{\prime}(0)+\lambda \phi(0)\right]=\frac{\sigma^{2}}{9 m_{0}}+\cdots,
$$

a power series in $\lambda$ in which the coefficients are power series in $e$. The deter-

* Moulton's Periodic Orbits, \& 18. 
minant $\Delta$ is different from zero for $e=\lambda=0$, and therefore remains different from zero for $|e|$ and $|\lambda|$ sufficiently small. Consequently the two solutions $\phi$ and $\chi+\lambda t \phi$ constitute a fundamental set and the general solution of $(14 b)$ is

$$
w=B_{1} \phi+B_{2}[\chi+\lambda t \phi],
$$

where $B_{1}$ and $B_{2}$ are the constants of integration.

\section{EXISTENCE OF SYMMETRICAL PERIODIC ORBITS}

Let us take the initial conditions

$$
p(0)=\alpha_{1}, \quad p^{\prime}(0)=0, \quad w(0)=0, \quad w^{\prime}(0)=\alpha_{2} .
$$

With these initial conditions it can be shown from the properties of the differential equations (13) that $p$ is even in $t$ and $w$ is odd in $t$. Hence sufficient conditions that $p$ and $w$ shall be periodic with the period $T$ are

$$
p^{\prime}\left(\frac{T}{2}\right)=0, \quad w\left(\frac{T}{2}\right)=0 .
$$

In order to establish the existence of symmetrical periodic orbits, we integrate (13) as power series in $\alpha_{1}, \alpha_{2}$, and $\mu$, subject to the initial conditions (25), and impose the periodicity conditions (26) upon the solutions. It will be shown that (26) can be satisfied by a determination of $\alpha_{1}$ and $\alpha_{2}$ as power series in $\mu$.

The differential equations from which the linear terms in $\alpha_{1}$ and $\alpha_{2}$ are obtained are the same as the equations of variation. The solutions of these equations which satisfy the initial conditions (25) are

$$
p=\frac{\alpha_{1}}{\theta(0)}\left[\theta+\gamma e^{2} t \psi\right], \quad w=\frac{\alpha_{2}[\chi+\lambda t \phi]}{\left[\chi^{\prime}(0)+\lambda \phi(0)\right]} .
$$

When the terms in $\mu$ and higher degree terms in $\mu, \alpha_{1}$, and $\alpha_{2}$ are taken in (13), the solutions satisfying (25) have the form

$$
\begin{aligned}
& p=\frac{\alpha_{1}}{\theta(0)}\left[\theta+\gamma e^{0} t \psi\right] \\
& + \text { terms in } \mu \text { and higher degree terms in } \mu, \alpha_{1} \text {, and } \alpha_{2} \text {, } \\
& w=\frac{\alpha_{2}[\chi+\lambda t \phi]}{\left[\chi^{\prime}(0)+\lambda \phi(0)\right]}
\end{aligned}
$$

+ terms in $\mu$ and higher degree terms in $\mu, \alpha_{1}$, and $\alpha_{2}$. 
After imposing the conditions (26) upon (27), we obtain

$$
0=\frac{\alpha_{1}}{\theta(0)}\left[\theta^{\prime}\left(\frac{T}{2}\right)+\gamma e^{2} \frac{T}{2} \psi^{\prime}\left(\frac{T}{2}\right)+\gamma e^{2} \psi\left(\frac{T}{2}\right)\right]
$$

+ terms in $\mu$ and higher degree terms in $\mu, \alpha_{1}$, and $\alpha_{2}$,

$$
\begin{aligned}
& 0=\frac{\alpha_{2}\left[\chi\left(\frac{T}{2}\right)+\lambda \frac{T}{2} \phi\left(\frac{T}{2}\right)\right]}{\chi^{\prime}+\lambda \phi(0)} \\
& + \text { terms in } \mu \text { and higher degree terms in } \mu, \alpha_{1} \text {, and } \alpha_{2} \text {. }
\end{aligned}
$$

From the form of $\psi, \theta$ and $\chi$ it follows that

$$
\psi\left(\frac{T}{2}\right)=\theta^{\prime}\left(\frac{T}{2}\right)=\chi\left(\frac{T}{2}\right)=0,
$$

and therefore the determinant of the linear terms in $\alpha_{1}$ and $\alpha_{2}$ in (28)

$$
\delta=-\frac{\gamma e^{2} \lambda T^{2} \psi^{\prime}\left(\frac{T}{2}\right) \phi\left(\frac{T}{2}\right) \psi^{\prime}(0) \phi(0)}{4 D \Delta} .
$$

The value of $\delta$ depends upon the parity of $\nu$ and $N$ in $T=2 \nu \pi=2 N \pi / \sigma$. The determinants $D$ and $\Delta$ are both different from zero for $|e|$ and $|\lambda|$ sufficiently small and are both finite. Hence $\delta$ can become zero only when the numerator vanishes. We shall show that the numerator of (29) is different from zero provided that $e$ and $\lambda$ are not zero but sufficiently small numerically.

Suppose $\nu$ is even, then $N$ is odd, since $\nu$ and $N$ are relatively prime, and from the form of $\psi$ and $\phi$ it follows that

$$
\psi^{\prime}\left(\frac{T}{2}\right)=\psi^{\prime}(0) \neq 0, \quad \phi\left(\frac{T}{2}\right)=-\phi(0) \neq 0 .
$$

If $\nu$ is odd then

$$
\psi^{\prime}\left(\frac{T}{2}\right)=-1+2 e+\cdots
$$

and

$\phi\left(\frac{T}{2}\right)=\frac{\sqrt{2}}{3 \sqrt{m_{0}}}+e[$ power series in $e]$ or $-\frac{\sqrt{2}}{3 \sqrt{m_{0}}}+e[$ power series in $e]$, according as $N$ is even or odd. Therefore $\delta$ is different from zero whatever be the parity of $\nu$ and $N$ provided $|e|$ and $|\lambda|$ are sufficiently small. Consequently equations (28) can be solved for $\alpha_{1}$ and $\alpha_{2}$ as power series in $\mu$ which vanish with $\mu$ and converge for $|\mu|$ sufficiently small. Since the initial 
values of $p$ and $w$ are power series in $\mu$, the solutions will have the form

$$
p=\sum_{i=1}^{\infty} p_{i} \mu^{i}, \quad w=\sum_{i=1}^{\infty} w_{i} \mu^{i}
$$

where each $p_{i}$ and $w_{i}$ is periodic with the period $T$. The constant $d$ which appears first in (11) enters these solutions as an arbitrary, but when a value is assigned to $d$ the solutions in (30) are unique.

\section{Direct construction of the symmetrical Periodic orbits}

Let us substitute (30) in (13) and equate the coefficients of the various powers of $\mu$. We thus obtain a series of differential equations which can be integrated step by step. The constants of integration appearing at each stage are to be determined so that the solutions shall be periodic and satisfy the symmetrical initial conditions

$$
p^{\prime}(0)=0, \quad w(0)=0 .
$$

When these symmetrical initial conditions are imposed on (30) we obtain

$$
p_{i}^{\prime}(0)=0, \quad w_{i}(0)=0 \quad(i=1, \cdots \infty) .
$$

The differential equations for the terms in $\mu$ are
(a) $p_{1}^{\prime \prime}+P(e, t) p_{1}=P^{(1)}$,
(b) $w_{1}^{\prime \prime}+W(\lambda, e, t) w_{1}=W^{(1)}$.

The left members of these equations are the same as the equations of variation. The right member $P^{(1)}$ is a $\lambda$-cosine-series and is independent of $w_{1}$. The right member of $(32 b)$ depends on $p_{1}$. The terms of $W^{(1)}$ which are independent of $p_{1}$ form a $\lambda^{1 / 2}$-sine-series and the remaining terms are also a $\lambda^{1 / 2}$-sine-series multiplied by $p_{1}$.

The complementary function of $(32 a)$ is

$$
p_{1}=a_{1}^{(1)} \psi+a_{2}^{(1)}\left[\theta+\gamma e^{2} t \psi\right],
$$

where $a_{1}^{(1)}$ and $a_{2}^{(1)}$ are the constants of integration. To obtain the particular integral we employ the method of the variation of parameters. By this method we have

$$
\begin{gathered}
\left(a_{1}^{(1)}\right)^{\prime} \psi+\left(a_{2}^{(1)}\right)^{\prime}\left[\theta+\gamma e^{2} t \psi\right]=0 \\
\left(a_{1}^{(1)}\right)^{\prime} \psi^{\prime}+\left(a_{2}^{(1)}\right)^{\prime}\left[\theta+\gamma e^{2}\left(t \psi^{\prime}+\psi\right)\right]=P^{(1)} .
\end{gathered}
$$

The determinant of the coefficients of $\left(a_{1}^{(1)}\right)^{\prime}$ and $\left(a_{2}^{(1)}\right)^{\prime}$ is $D$, the same as in (16), and is different from zero. Hence

$$
\left(a_{1}^{(1)}\right)^{\prime}=-\frac{\left[\theta+\gamma e^{2} t \psi\right] P^{(1)}}{D}, \quad\left(a_{2}^{(1)}\right)^{\prime}=\frac{\psi P^{(1)}}{D} .
$$


When these equations are integrated and the results are substituted in (33), we obtain the solution

$$
p_{1}=A_{1}^{(1)} \psi+A_{2}^{(1)}\left[\theta+\gamma e^{2} t \psi\right]+C^{(1)}-t \psi\left[L_{0}^{(1)}+\lambda^{\nu / 2} e^{N} L_{1}^{(1)} \text { or } \lambda^{\nu} e^{2 N} L_{2}^{(1)}\right]
$$

according as $\nu$ is even or odd respectively. The terms in (36) not already defined have the following properties: $A_{1}^{(1)}$ and $A_{2}^{(1)}$ are the constants of integration; $C^{(1)}$ is a $\lambda$-cosine-series; $L_{1}^{(1)}$ and $L_{2}^{(1)}$ are power series in $\lambda$ with coefficients which are constant power series in $e$, and $L_{v}^{(1)}$ is a power series in $e$ with constant coefficients. In order that $p_{1}$ shall satisfy (31) and shall be periodic, the constants of integration must have the values

$$
A_{1}^{(1)}=0, \quad A_{2}^{(1)}=\frac{1}{\gamma e^{2}} L_{0}^{(1)}+\frac{1}{\gamma}\left[\lambda^{\nu / 2} e^{N-2} L_{1}^{(1)} \text { or } \quad \lambda^{\nu} e^{2 N-2} L_{2}^{(1)}\right],
$$

according as $\nu$ is even or odd. Hence the solution of $(32 a)$ which is periodic and satisfies (31) is

$$
p_{1}=\frac{1}{e^{2}} C_{1}(t),
$$

where $C_{1}(t)$ is a $\lambda$-cosine-series.

With $p_{1}$ determined as in (38), the right member of $(32 b)$ takes the form of $a \lambda^{1 / 2}$-sine-series multiplied by $1 / e^{2}$. The complete solution of $(32 b)$ is obtained in the same way as (36) was obtained. The complementary function of $(32 b)$ is

$$
w_{1}=b_{1}^{(1)} \phi+b_{2}^{(1)}[\chi+\lambda t \phi],
$$

and the equations analogous to (34) are

$$
\begin{gathered}
\left(b_{1}^{(1)}\right)^{\prime} \phi+\left(b_{2}^{(1)}\right)^{\prime}[\chi+\lambda t \phi]=0, \\
\left(b_{1}^{(1)}\right)^{\prime} \phi^{\prime}+\left(b_{2}^{(1)}\right)^{\prime}\left[\chi^{\prime}+\lambda\left(t \phi^{\prime}+\phi\right)\right]=W^{(1)} .
\end{gathered}
$$

The determinant of the coefficients of $\left(b_{1}^{(1)}\right)^{\prime}$ and $\left(b_{2}^{(1)}\right)^{\prime}$ is $\Delta$, the same as in (23), and is different from zero. Hence

$$
\left(b_{1}^{(1)}\right)^{\prime}=-\frac{[\chi+\lambda t \phi] W^{(1)}}{\Delta}, \quad\left(b_{2}^{(1)}\right)^{\prime}=\frac{\phi W^{(1)}}{\Delta} .
$$

When these equations are integrated and the results are substituted in (39), the general solution of $(32 b)$ is found to be

$$
w_{1}=B_{1}^{(1)} \phi+B_{2}^{(1)}[\chi+\lambda t \phi]+\frac{1}{e^{2}} S^{(1)}-\frac{\lambda^{1 / 2}}{e^{2}} M^{(1)} t \phi,
$$

where $B_{1}^{(1)}$ and $B_{2}^{(1)}$ are the constants of integration; $S^{(1)}$ is a $\lambda^{1 / 2}$-sine-series, and $M^{(1)}$ has the same form as $L_{1}^{(1)}$ or $L_{2}^{(1)}$ in (36). In order that $w_{1}$ shall satisfy the initial conditions (31) and be periodic, the constants of integration 
must have the values

$$
B_{1}^{(1)}=0, \quad B_{2}^{(1)}=\frac{M^{(1)}}{e^{2} \lambda^{1 / 2}} .
$$

Hence the desired solution of $(32 b)$ is

$$
w_{1}=\frac{1}{e^{2} \lambda} S_{1}(t)
$$

where $S_{1}(t)$ is a $\lambda^{1 / 2}$-sine-series.

The remaining steps of the integration can be carried on in the same way, and the solutions are almost entirely similar to those obtained at the first step. The presence of the terms in $1 / e^{2}$ and $1 / e^{2} \lambda$ in (38) and (43) introduces some slight changes in the forms of the solutions, and it is necessary to consider the differential equations up to the terms in $\mu^{3}$ in order to discover the law according to which the remaining solutions proceed.

The terms in $\mu^{2}$ vield the differential equations
(a) $p_{2}^{\prime \prime}+P(e, t) p_{2}=P^{(2)}$
(b) $w_{2}^{\prime \prime}+W(\lambda, e, t) w_{2}=W^{(2)}$.

The right member of $(44 a)$ is a $\lambda$-cosine-series multiplied by $1 / e$, and the complete solution of the equation is

$$
p_{2}=A_{1}^{(1)} \psi+A_{2}^{(2)}\left[\theta+\gamma e^{2} t \psi\right]+\frac{1}{e^{2}} C^{(2)}-\frac{1}{e^{2}} L^{(2)} t \psi,
$$

where $A_{1}^{(2)}$ and $A_{2}^{(2)}$ are the constants of integration; $C^{(2)}$ is a $\lambda$-cosine-series; and $L^{(2)}$ is a power series in $\lambda$ with coefficients which are constant power series in $e$. The numerical values of the coefficients in $L^{(2)}$ vary with the parity of $\nu$, but the series has the same form whether $\nu$ is even or odd. In order that $p_{2}$ shall be periodic and satisfy the conditions (31) the constants of integration must have the values

$$
A_{1}^{(2)}=0, \quad A_{2}^{(2)}=\frac{L^{(2)}}{e^{4}},
$$

and the desired solution for $p_{2}$ then takes the form

$$
p_{2}=\frac{1}{e^{4}} C_{2}(t)
$$

where $C_{2}(t)$ is a $\lambda$-cosine-series.

The right member of $(44 b)$ is completely known when $p_{2}$ has been determined and is a $\lambda^{1 / 2}$-sine-series multiplied by $1 / e^{4} \lambda$. The complete solution of $(44 b)$ is readily found to be

$$
w_{2}=B_{1}^{(2)} \phi+B_{2}^{(2)}[\chi+\lambda t \phi]+\frac{1}{e^{4} \lambda} S^{(2)}-\frac{1}{e^{4} \lambda^{1 / 2}} M^{(2)} t \phi,
$$

where the undefined terms in (47) denote functions similar to those represented by the corresponding terms in (41). If the initial and periodicity conditions 
are to be satisfied then

$$
B_{1}^{(2)}=0, \quad B_{2}^{(2)}=\frac{M^{(2)}}{e^{4} \lambda^{3 / 2}} .
$$

Hence the desired solution for $w_{2}$ is

$$
w_{2}=\frac{1}{e^{4} \lambda^{2}} S_{2}(t),
$$

where $S_{2}(t)$ is a $\lambda^{1 / 2}$-sine-series.

The differential equations obtained from the terms in $\mu^{3}$ are
(a) $p_{3}^{\prime \prime}+P(e, t) p_{3}=P^{(3)}$
(b) $u_{3}^{\prime \prime}+W(\lambda, e, t) u_{3}=W^{(3)}$.

The right member $P^{(3)}$ is a $\lambda$-cosine-series multiplied by $1 / e^{4} \lambda$, and the complete solution of $(48 a)$ is

$$
p_{3}=A_{1}^{(3)} \psi+A_{2}^{(3)}\left[\theta+\gamma e^{2} t \psi\right]+\frac{1}{e^{4} \lambda} C^{(3)}-\frac{1}{e^{4} \lambda} L^{(3)} t \psi,
$$

where the undefined terms denote functions similar to those represented by the corresponding terms in (45). If the initial and periodicity conditions are to be satisfied, then

$$
A_{1}^{(3)}=0, \quad A_{2}^{(3)}=\frac{1}{\gamma e^{6} \lambda} L^{(3)},
$$

and the desired solution for $p_{3}$ becomes

$$
p_{3}=\frac{1}{e^{6} \bar{\lambda}} C_{3}(t)
$$

where $C_{3}(t)$ is a $\lambda$-cosine-series.

From the forms of $p_{3}$ and $w_{2}$ it follows that $W^{(3)}$ is a $\lambda^{1 / 2}$-sine-series multiplied by $1 / e^{6} \lambda^{2}$. Hence the solution for $w_{3}$ will be a $\lambda^{1 / 2}$-sine-series multiplied by $1 / e^{6} \lambda^{3}$. By an induction to the general term it can readily be shown that $p_{j}$ and $w_{j}$ have the forms

$$
\begin{array}{ll}
p_{j}=\frac{1}{e^{2 j} \lambda^{j-2}} C_{j}(t) & (j=2,3, \cdots \infty), \\
w_{j}=\frac{1}{e^{2 j} \lambda^{j}} S_{j}(t) & (j=1,2, \cdots \infty),
\end{array}
$$

where each $C_{j}(t)$ is a $\lambda$-cosine-series and each $S_{j}(t)$ is a $\lambda^{1 / 2}$-sine-series.

7. Proof that all periodic orbits Represented By POWER SERIES IN $\mu$ ARE SYMME TRICAL

The usual method of proving that all periodic orbits are symmetrical is to assume the initial conditions

$$
p(0)=\alpha_{1}, \quad p^{\prime}(0)=\alpha_{2}, \quad w(0)=\alpha_{3}, \quad w^{\prime}(0)=0,
$$


and integrate equations (13) as power series in $\alpha_{i}(i=1,2,3)$ and $\mu$. Sufficient conditions that the solutions shall be periodic are

$$
\begin{gathered}
p(T)-p(0)=0, \quad p^{\prime}(T)-p^{\prime}(0)=0, \quad w(T)-w(0)=0, \\
w^{\prime}(T)-w^{\prime}(0)=0 .
\end{gathered}
$$

If there exists an integral of the differential equations (5) it could be shown by means of this integral that the last condition of (50) would be reduntant. Then the three independent conditions of (50) could be satisfied by a proper determination of $\alpha_{i}(i=1,2,3)$ as power series in $\mu$ provided a certain determinant did not vanish. Thus periodic solutions would be shown to exist and they would be unique for any assigned value of $d$ which would enter the solutions through (11). Since the symmetrical solutions are also unique under the same circumstance and since the general orbits having the initial conditions (49) would include those which are symmetrical, all the solutions would therefore be symmetrical.

In the problem under consideration no integral of equations (5) is known to exist and we can not use the foregoing method. We show, however, from the construction of the solutions that the symmetrical initial conditions are a consequence of the periodicity conditions and therefore that all the orbits represented by solutions which are power series in $\mu$ with periodic coefficients are symmetrical.

If the symmetrical initial conditions (31) are not imposed upon (36), the periodic solution for $p_{1}$ is

$$
p_{1}=A_{1}^{(1)} \psi+C_{1}(t) .
$$

When this value of $p_{1}$ is substituted in the right member of the differential equations defining $w_{1}$ we obtain

$$
u_{1}^{\prime \prime}+W(\lambda, e, t) w_{1}=W^{(1)}+A_{1}^{(1)} \bar{W}^{(1)},
$$

where $\bar{W}^{(1)}$ is similar to a $\lambda^{1 / 2}$-sine-series except that it has cosines instead of sines. The complete solution of this equation is the same as (41) with the additional terms

$$
A_{1}^{(1)}\left[\text { periodic terms }+ \text { terms in } t^{2} \phi \text { and } t \chi\right] .
$$

The only way in which this solution can be made periodic is by putting $A_{1}^{(1)}=0$ and then determining $B_{2}^{(1)}$ as in (42). Hence the periodic solution for $w_{1}$ which does not satisfy the condition $w_{1}(0)=0$ becomes

$$
w_{1}=B_{1}^{(1)} \phi+\frac{1}{e^{2} \lambda} S_{1}(t),
$$

where $B_{1}^{(1)}$ remains undetermined at this step. In order to obtain the value 
of $B_{1}^{(1)}$ we must consider the differential equation which defines $p_{2}$. It has the form

$$
p_{2}^{\prime \prime}+P(e, t) p_{2}=P^{(2)}+B_{1}^{(1)} \bar{P}^{(2)},
$$

where $\bar{P}^{(2)}$ is similar to a $\lambda^{1 / 2}$-sine-series except that it has even multiples of $\sigma$ instead of odd. The complete solution of (52) is the same as (45) with the additional terms

$$
B_{1}^{(1)}\left[\text { periodic terms }+ \text { terms in } t^{2} \psi \text { and } t \theta\right] .
$$

The only way in which $p_{2}$ can be made periodic is by putting $B_{1}^{(1)}=0$ and then determining $A_{2}^{(2)}$ in the same way as in (46).

Thus the integrations of the differential equations arising at each step can be obtained in the same way and the process continued as far as desired. The constant of integration which is not determined by the condition that $p_{j}$ shall be periodic must be zero in order that $w_{j}$ shall be periodic; and similarly the constant of integration which is not determined by the condition that $w_{j}$ shall be periodic must be zero in order that $p_{j+1}$ shall be periodic. The solutions thus obtained are the same as those obtained for the symmetrical orbits. Hence the symmetrical initial conditions (31) are a consequence of the periodicity conditions and all periodic orbits represented by power series in $\mu$ are symmetrical.

\section{INTEGRATION OF EQUATION (7)}

There remains one differential equation which has not been integrated, viz.,

$$
r^{2} v^{\prime}=c .
$$

In order to integrate this equation we make in (7) the substitutions

$$
v=v_{0}+q, \quad r=r_{0}+p, \quad c=c_{0}+d \mu,
$$

where $q$ vanishes with $\mu$, and $p$ has the solution obtained in $\S 6$. Then equation (7) becomes

$$
q^{\prime}=Q_{1} \mu+Q_{2} \mu^{2}+\cdots+Q_{j} \mu^{j}+\cdots,
$$

where each $Q_{j}$ is similar in form to $p_{j}$, that is, each $Q_{j}$ is a $\lambda$-cosine-series multiplied by $1 / e^{2 j} \lambda^{j-2}$. As the right side of (54) contains constant terms, the integration will yield terms in $t$ unless these terms can be made to vanish. Now the constant $d$ appears in these terms but as the constant part of the coefficient of $\mu$ does not vanish when $d=0$, it is impossible to determine $d$ so as to eliminate the constant terms in (54). Hence the integration of (54) gives

$$
q=\sum_{j=1}^{\infty}\left[q_{j}+t \bar{q}_{j}\right] \mu^{j}+q_{0}
$$


where each $q_{j}$ is similar in form to $Q_{j}$ except that it has sines instead of cosines, each $\bar{q}_{j}$ is similar to $Q_{j}(0)$, and $q_{0}$ is the constant of integration. Since the equal masses are started from the $\zeta$-axis at the initial time then $v(0)=0$. Since $v_{0}(0)=0$ it follows that $q(0)=0$ and as each $q_{j}(0)=0$ then $q_{0}=0$.

This completes the integration of the differential equations defining the motion of the three finite bodies $m_{1}, m_{2}$, and $\mu$. The constant of integration arising from the integral of areas remains undetermined, but the remaining constants of integration can be determined by the periodicity conditions alone. The mass $\mu$ is restricted in magnitude because of certain convergence conditions but it can be increased step by step by making the analytical continuation of the solutions already obtained, provided the series do not pass through any singularities in the intervals. This can be done by the method already developed.

QUeen's University, Kingston, Ontario, August 17, 1914. 